\title{
Perfil da farmacoterapia utilizada por idosos institucionalizados
}

\author{
Profile of the pharmacotherapy used by institutionalized elderly \\ Perfil de la farmacoterapia utilizada por ancianos asilados \\ Cléa Adas Saliba GARBIN ${ }^{1}$ \\ Thaís Jaqueline Vieira de LIMA ${ }^{1}$ \\ Paula Caetano ARAÚJO ${ }^{2}$ \\ Artênio José Ísper GARBIN ${ }^{1}$ \\ Renato Moreira ARCIERI ${ }^{1}$ \\ Orlando SALIBA ${ }^{1}$ \\ ${ }^{I}$ Departamento de Odontologia Infantil e Social, Faculdade de Odontologia de Araçatuba, Universidade Estadual Paulista \\ (UNESP), 16015-050, Araçatuba-SP, Brasil \\ ${ }^{2}$ Área de Odontologia Preventiva e Social, Faculdade de Odontologia da Universidade Federal de Uberlândia (FOUFU), \\ 38405320, Uberlândia-MG, Brasil
}

\begin{abstract}
Resumo
Introdução: O desenvolvimento de patologias crônicas aumenta com o avançar da idade, bem como a quantidade de medicamentos administrados, desafiando o Sistema Único de Saúde quanto a polifarmácia e a farmacovigilância. Objetivos: Este estudo objetivou verificar o perfil da farmacoterapia utilizada por idosos institucionalizados, através da identificação dos medicamentos mais usados, da detecção da ocorrência da polifarmácia e do uso de medicamentos inapropriados para idosos. Material e Método: Os dados foram obtidos através de consulta aos prontuários médicos dos idosos e entrevistas semi-estruturadas às enfermeiras responsáveis de seis Instituições de Longa Permanência para Idosos de quatro municípios do estado de São Paulo. A polifarmácia foi definida através do uso concomitante de cinco ou mais medicamentos diários, sendo que os medicamentos potencialmente inapropriados para idosos foram identificados através dos critérios de Beers. Também foi verificado os fatores associados ao uso de MPI, por meio de análise multivariada. Resultados: Participaram do estudo 261 idosos com idade $\geq 60$ anos, sendo que 73,9\% foram acometidos pela polifarmácia. Foram prescritos 1452 medicamentos, sendo a maioria (36\%) com ação sobre o Sistema Nervoso Central. 50,6\% consumiam pelo menos um MPI diariamente, sendo o diazepam $(8,4 \%)$ e a fluoxetina $(7,7 \%)$ os mais utilizados. Conclusão: Desta forma, a polifarmácia se faz presente entre a maioria dos idosos, havendo uma prevalência de antipsicóticos e antidepressivos. Quanto ao uso de medicamentos potencialmente inapropriados verificou-se uma alta prevalência, sendo a polifarmácia e a depressão os fatores associados a sua utilização.
\end{abstract}

Descritores: Uso Indevido de Medicamentos sob Prescrição; Idoso; Prescrição Inadequada.

\begin{abstract}
Introduction: The development of chronic pathologies increases with age, as well as the quantity of drugs administered, challenging the Brazilian public health system regarding polypharmacy and pharmacovigilance. Objectives: This study aimed to verify the profile of pharmacotherapy used by institutionalized elderly, through the identification of the most used drugs, the detection of the occurrence of polypharmacy and the use of inappropriate drugs for elderly. Material and Method: The datas were obtained by consulting the medical records of elderly and applying semi-structured interviews with the responsible nurses of six long-stay institutions for elderly in four cities of São Paulo State, Brazil. Polypharmacy was defined as the concomitant use of five or more daily medications; while potentially inappropriate medications for elderly were identified using the Beers criteria. The factors associated with the use of potentially inappropriate medications were performed by multivariate analysis. Results: The study included 261 elderly aged $\geq 60$ years old, being 73,9\% affected by polypharmacy. 1452 medications were prescribed, where most of them $(36 \%)$ act on the central nervous system. $50,6 \%$ of the patients consumed at least one potentially inappropriate medications daily, being diazepam $(8,4 \%)$ and fluoxetine $(7,7 \%)$ the most widely used. Conclusion: Therefore, polypharmacy is present among most elderly, with a prevalence of anti-psychotics and antidepressants. There was a high prevalence regarding the use of potentially inappropriate medications, being polypharmacy and depression the factors associated with its utilizacion.
\end{abstract}

Descriptors: Prescription Drug Misuse; Aged; Inappropriate Prescribing.

\section{Resumen}

Introducción: El desarrollo de enfermedades crónicas aumenta con la edad avanzada, bien como la cantidad de fármacos administrados, desafiando lo Sistema Único de Salud cuanto la polifarmacia y la farmacovigilancia. Objetivos: Este estudio tuvo como objetivo determinar el perfil de la farmacoterapia utilizada por ancianos institucionalizados, mediante la identificación de fármacos más usados, de la detección de la polifarmacia y del uso de medicamentos inapropiados para ancianos. Material y Método: Los datos fueron obtenidos mediante consulta a los registros médicos de los ancianos y entrevistas semiestructuradas a las enfermeras responsables de seis Instituciones de Larga Estadía para las Personas Mayores de cuatro municipios del estado de Sao Paulo. La polifarmacia fue definida por el uso concomitante de cinco o más medicamentos al día, y los medicamentos potencialmente inapropiados para ancianos fueron identificados por los criterios de Beers. También fueron verificados los factores asociados con el uso de medicamentos potencialmente inapropiados, por el análisis multivariante. Resultados: El estudio incluyó 261 ancianos con edades $\geq 60$ años, siendo que $73,9 \%$ fueron afectados por la polifarmacia. 1452 medicamentos fueron prescritos, siendo la mayoría (36\%) con acción sobre el Sistema Nervioso Central. 50,6\% consumieron al menos un medicamento potencialmente inapropiados diario, siendo lo diazepam $(8,4 \%)$ y la fluoxetina $(7,7 \%)$ los más utilizados. Conclusión: Por lo tanto, la polifarmacia está presente entre la mayoría de los ancianos, habiendo prevalencia de los antipsicóticos y antidepresivos. En relación al uso de medicamentos potencialmente inapropiados, fue verificada una alta prevalencia, siendo la polifarmacia y la depresión los factores asociados con su utilización.

Descriptores: Mal Uso de Medicamentos de Venta con Receta; Anciano; Prescripción Inadecuada.

\section{INTRODUÇÃO}

Os idosos constituem um segmento da população cuja tendência a consumir medicamentos somada à interferência das alterações fisiopatológicas inerentes ao envelhecimento, podem levá-los a sofrer graves reações adversas, e estas, por sua vez, implicam em sérias consequências como internações hospitalares, e até mesmo o óbito ${ }^{1}$.

Frente a isso, têm sido desenvolvidos instrumentos visando detectar potenciais riscos de iatrogenia 
medicamentosa em idosos, sendo os Critérios de Beers ${ }^{-4}$, ao longo de 20 anos, o mais consultado quando se trata de avaliar a prescrição medicamentosa de idosos ${ }^{5,6}$.

Beers $^{3}$, em 1997, estabeleceu critérios, baseados em trabalhos publicados sobre medicamentos e farmacologia do envelhecimento, para definir uma lista de fármacos potencialmente inadequados para idosos ${ }^{5}$, entre os quais podem ser citados os benzodiazepínicos e os hipoglicemiantes orais de meia vida longa; os barbituratos de curta duração; os antidepressivos com forte ação anticolinérgica; os analgésicos opioides; as associações em doses fixas de antidepressivos e antipsicóticos; alguns relaxantes musculares, como a orfenadrina e o carisoprodol ${ }^{4}$.

A preocupação com o uso de medicamentos potencialmente inapropriados (MPI) para idosos levou ao desenvolvimento de vários estudos, nos mais diversos cenários de atenção à saúde, e apesar do vasto repertório de informações sobre o assunto, muitos MPI continuam sendo prescritos e utilizados como tratamento de primeira escolha para idosos, o que evidencia a necessidade de mais trabalhos sobre o assunto $^{7}$, visto que o uso de MPI tem sido intrinsecamente associado à ocorrência de reações adversas a medicamentos e suas consequências ${ }^{8,9}$.

Chrischilles e colaboradores ${ }^{10}$ (2009) afirmam que o principal agente que impulsiona a ocorrência de reações adversas é o número de medicamentos consumidos por este segmento da população, que, por conseguinte, reflete uma maior prevalência de patologias crônicas e múltiplas. Assim, verifica-se a importância de verificar também a quantidade de medicamentos utilizados individualmente, sendo o uso de cinco ou mais medicamentos utilizados concomitantemente conhecido como polifarmácia ${ }^{11,12}$

A polifarmácia pode ser considerada uma terapêutica personalizada, desde que o médico prescreva somente medicamentos necessários para a patologia que o paciente apresenta. Isto, além de evitar os efeitos colaterais de componentes desnecessários, reduz o custo do tratamento ${ }^{13}$.

$\mathrm{O}$ aprimoramento da qualidade da prescrição médica ao grupo em questão e o estímulo às investigações sobre o uso de medicamentos entre indivíduos institucionalizados devem beneficiar não somente essa população, mas todos os idosos ${ }^{14}$. Além disso, idosos institucionalizados não são frequentemente incluídos em estudos farmacoepidemiológicos, apesar de constituírem grupos de idosos com acentuada utilização de medicamentos ${ }^{15}$.

Dessa forma, o objetivo deste estudo foi analisar o perfil da farmacoterapia utilizada por idosos institucionalizados de quatro municípios do estado de São Paulo. Esta análise foi realizada por meio da identificação dos medicamentos mais frequentemente utilizados, da detecção da ocorrência da polifarmácia, uso de medicamentos potencialmente inapropriados para uso em idosos e os fatores associados à sua ocorrência, visando contribuir com um melhor conhecimento da terapia medicamentosa utilizada por residentes em Instituições de Longa Permanência para Idosos (ILPI), e ao mesmo tempo, oferecer condições para o desenvolvimento de novas estratégias aos serviços de saúde para o aprimoramento da qualidade da assistência farmacêutica ao idoso.

\section{MATERIAL E MÉTODO}

\section{o Desenho do Estudo}

Trata-se de um estudo transversal cuja população constituiu-se de idosos com idade igual ou superior a 60 anos, residentes em seis ILPI públicas de quatro municípios do estado de São Paulo (Araçatuba, Bauru, Botucatu, e Penápolis).

Os dados foram coletados no período de dezembro de 2011 a maio de 2012, através da análise dos prontuários médicos dos idosos complementados por entrevista às enfermeiras responsáveis de cada ILPI, visto que os idosos envolvidos na pesquisa apresentavam diferentes níveis de capacidade e graus variados de dependência. Desta forma, as informações solicitadas não poderiam ser colhidas de forma mais fidedigna senão pelo registro dos prontuários $\mathrm{e}$ entrevistas com as enfermeiras que acompanham os idosos constantemente.

Para a entrevista utilizou- se um questionário estruturado com perguntas abertas e fechadas, previamente testado, realizado por um pesquisador devidamente treinado.

Foram registrados os seguintes dados: características socioeconômicas (idade, renda familiar, escolaridade) e questões relacionadas à saúde, como internações hospitalares, patologias, sintomas ou síndromes presentes, bem como o uso diário de medicamentos prescritos (nome e indicação do medicamento). Também foram anotados os dados para avaliação do grau de dependência, que foi realizado através do índice de Katz ${ }^{16}$.

Foram catalogados todos os produtos farmacêuticos prescritos ao longo do mês anterior à coleta dos dados, onde registrou-se todas as informações do prontuário, incluindo as papeletas de prescrição diária e os receituários de cada residente.

Os medicamentos foram classificados em categorias terapêuticas, de acordo com seu princípio ativo, tendo como referência o Anatomical-Therapeutical-Chemical Classification System (ATC) adotado pela World Health Organization (WHO) ${ }^{17}$.

Os produtos em uso, com mais de um princípio ativo, foram enquadrados na categoria terapêutica do principal princípio; produtos com diferentes ações farmacológicas foram classificados levando-se em consideração a condição para a qual foram prescritos ou utilizados, como descritos por Coelho Filho et al. ${ }^{18}$.

A determinação de polifarmácia foi realizada mediante a contagem dos medicamentos usados concomitantemente, utilizando o ponto de corte de cinco ou mais medicamentos, de acordo com Klarin et al. ${ }^{11}$ e Pizzol et al. ${ }^{12}$.

Os medicamentos potencialmente inapropriados para idosos, ou seja, todos aqueles que deveriam ser evitados (independente de dose, duração do tratamento ou circunstâncias clínicas), tanto por não serem efetivos, como por apresentarem risco desnecessariamente alto para pessoas idosas (risco excedendo beneficio) ${ }^{18}$, foram avaliados através dos critérios de Beers ${ }^{4}$.

Além da prevalência do uso de MPI, verificou-se também quais fatores estavam associados à sua utilização, constituindo-se como variável dependente para fins de análise estatística o uso de pelo menos um medicamento inapropriado para idosos.

\section{- Variáveis Independentes}

As variáveis sociodemográficas e as variáveis relacionadas à saúde obtidas foram categorizadas para os propósitos das análises estatísticas. A idade foi categorizada em: $\geq 75$ e $<75$ e as internações hospitalares em: sim/não, se houve ou não, respectivamente, internação do indivíduo durante $\mathrm{o}$ ano anterior à pesquisa.

A polimorbidade foi identificada quando o paciente apresentava quatro ou mais doenças ${ }^{19}$, sendo categorizada 
em: sim, apresenta polimorbidade ( $\geq 4$ doenças) e não apresenta polimorbidade ( 0 a 3 doenças). As doenças verificadas nos prontuários médicos dos idosos foram agrupadas de acordo com a Classificação Internacional de Doenças, $10^{\mathrm{a}}$ revisão (CID-10) ${ }^{20}$.

O grau de dependência dos idosos foi medido pela escala de Katz ${ }^{16}$, sendo categorizado em: "dependente" (idosos classificados como "dependente" ou "parcialmente dependente" pela escala) e "não-dependente" (idosos classificados como "independente" pela escala ).

A determinação de polifarmácia foi realizada mediante a contagem dos medicamentos usados concomitantemente, utilizando o ponto de corte de cinco ou mais medicamentos, de acordo com outros autores ${ }^{11,12}$.

Os "distúrbios psiquiátricos", as "doenças cerebrovasculares" e a "depressão" também foram selecionados como variáveis independentes para compor a análise.

Foi considerado portador de distúrbio psiquiátrico, o idoso com diagnóstico de esquizofrenia, oligofrenia ou Mal de Alzheimer. Já as doenças cerebrovasculares incluíam: epilepsia, sequela de AVC, paraplegia e a atrofia dos membros.

Foram classificados como portadores de depressão os idosos que tiveram esse diagnóstico pela equipe médica da instituição e faziam tratamento para esta patologia.

○ Variáveis Independentes

Os dados foram sumarizados por análise descritiva, apresentados por distribuição absoluta e percentual.

Análises univariadas foram realizadas para avaliação isolada do efeito de cada variável. P-valores foram gerados utilizando o teste de associação Qui-quadrado e exato de Fischer.

A análise multivariada (regressão logística múltipla) foi conduzida para identificar fatores que se correlacionavam com o uso de medicamentos potencialmente inapropriados (MPI) para idosos. Foram selecionadas para constituir o modelo de regressão as variáveis que apresentaram valor de p <0,20 na análise univariada. Na análise multivariada, foram consideradas variáveis significantes aquelas com $\mathrm{p}<0,05$. Todas as análises foram conduzidas utilizando o programa estatístico SPSS versão 19.

- Aspectos Éticos

Este estudo foi aprovado pelo Comitê de Ética em Pesquisa da Faculdade de Odontologia de Araçatuba Universidade Estadual Paulista "Júlio de Mesquita Filho" UNESP - protocolo $\mathrm{n}^{\circ}$. 01988/2011, de acordo com a Resolução $n^{\circ}$. 196/1996 do Conselho Nacional de Saúde ${ }^{21}$. O Termo de Consentimento Livre e Esclarecido foi assinado pelos diretores das instituições, visto que os idosos envolvidos apresentavam níveis diferentes de comprometimento mental e graus variados de dependência, como explicado anteriormente.

\section{RESULTADOS}

Participaram do estudo 261 idosos, havendo predominância do gênero masculino $(57.5 \%)$, com idade avançada (igual ou superior a 75 anos) e comprometimento parcial ou total.

A maioria dos idosos $(67,8 \%)$ eram acometidos por múltiplas patologias (polimorbidade), sendo as doenças cerebrovasculares $(79,3 \%)$, a depressão $(66,7 \%)$ e os distúrbios psiquiátricos $(53,3 \%)$ as enfermidades mais frequentemente apresentadas.

Quanto ao uso de medicamentos, $96,9 \%$ faziam uso contínuo de medicamentos. Destes, 73,9\% consumiam diariamente mais de 4 medicamentos, caracterizando o fenômeno conhecido como polifarmácia. 1452 medicamentos foram prescritos aos idosos do presente estudo, sendo a maioria (36\%) com ação sobre o Sistema Nervoso Central (SNC).

A Figura 1 mostra os medicamentos mais utilizados pelos idosos, classificados segundo o sistema no organismo em que ocorre a ação terapêutica medicamentosa.

Com relação ao uso de medicamentos inapropriados para idosos, 50,6\% dos idosos consumiam no mínimo um MPI por dia, sendo o diazepam $(8,4 \%)$ e a fluoxetina $(7,7 \%)$ os mais frequentemente prescritos dentre a lista de medicamentos que devem ser evitados em idosos (ver Tabela 1). Cabe aqui salientar que entre os idosos que faziam uso de MPI, 72\% consumiam um MPI, 29\% receberam 2 MPIs e $6 \%$ consumiam 3 ou mais MPI simultaneamente.

Figura 1: Distribuição percentual dos idosos em relação ao uso de medicamentos, classificados de acordo com o local de ação terapêutica.

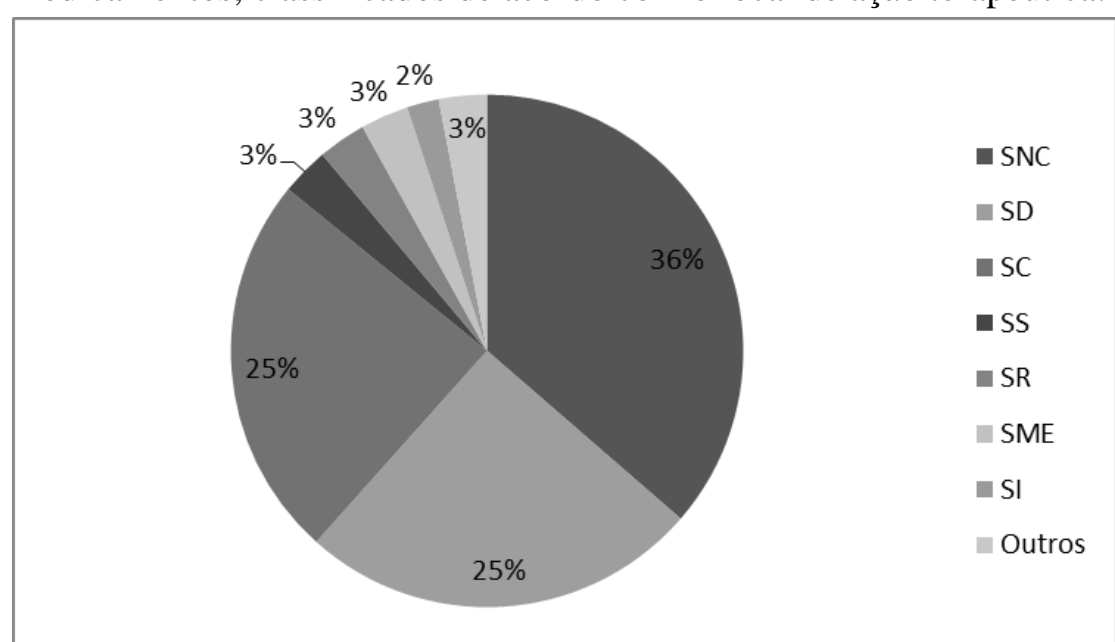

Legenda: SNC = Sistema Nervoso Central; $\mathrm{SD}=$ Sistema Digestivo $\mathrm{SC}=$ Sistema Cardiovascular; SS = Sistema Sanguíneo; $\mathrm{SR}=$ Sistem Respiratório; SME = Sistema Músculo-Esquelético; SI - Sistema Imunológico.

Tabela 1. Prevalência de MPI para idosos, independentemente do diagnóstico ou da condição clínica

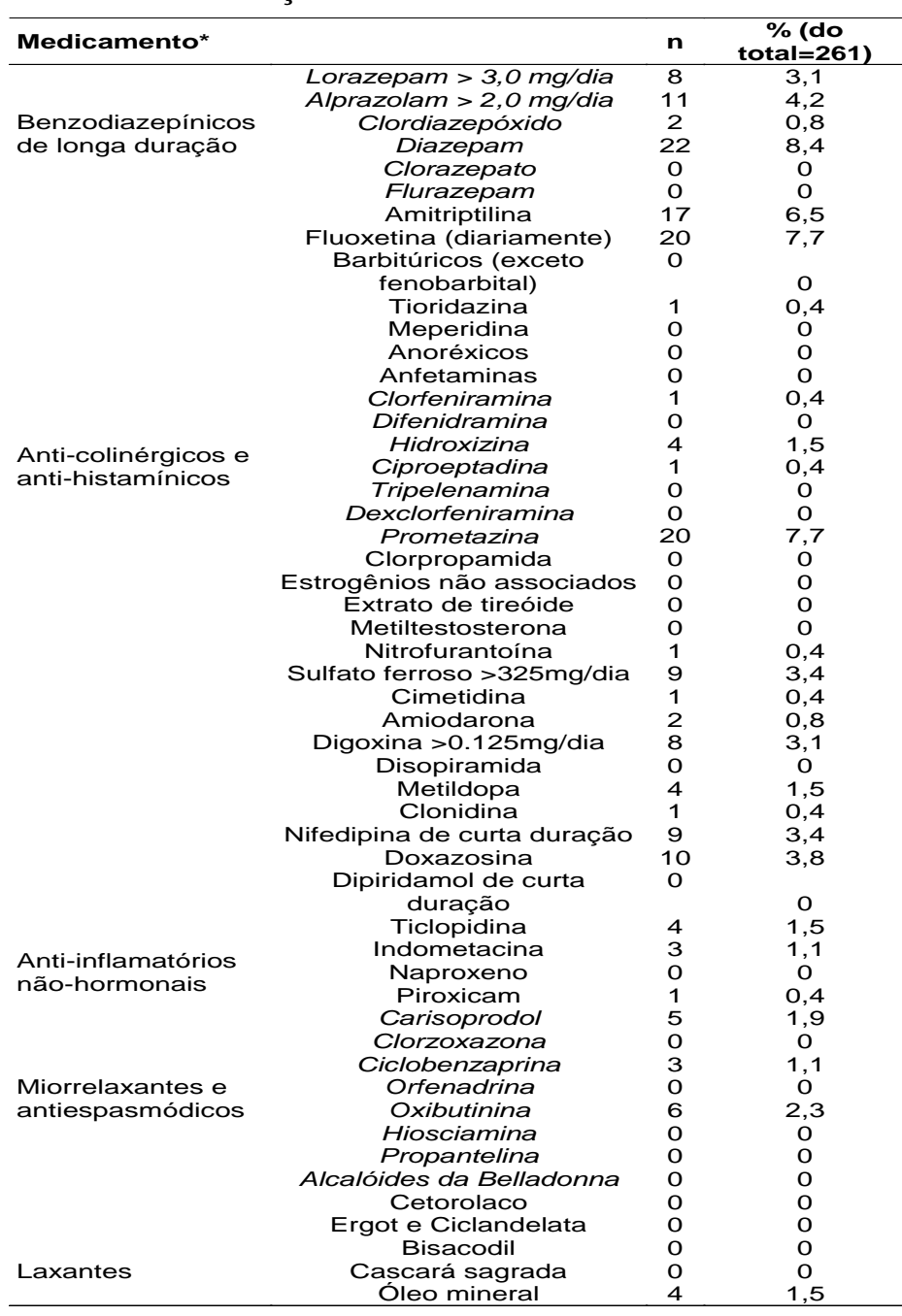

*Medicamentos que devem ser evitados por idosos, independentemente do diagnóstico ou da condição clínica, devido a alto risco de efeitos colaterais e com opcões à prescrição de outros fármacos pelos critérios de Beers-Fick (Fick et al ${ }^{4}$ ) e comercializados no Brasil (Gorzoni et al ${ }^{5}$ ). 
A análise univariada mostrou que polifarmácia, polimorbidade e depressão foram significativamente mais presentes em idosos tratados com MPI comparados com os idosos que não consumiam esse tipo de medicamento (Tabela 2).

Tabela 2. Análise univariada das variáveis que influenciaram o uso de Medicamentos Potencialmente Inapropriados.

\begin{tabular}{|c|c|c|c|c|c|c|c|c|}
\hline Variáveis & $\begin{array}{c}\text { Coeficiente } \\
\text { (B) }\end{array}$ & $\begin{array}{c}\text { Erro } \\
\text { Padrão }\end{array}$ & Wald & G.L* & p valor ${ }^{\star \star}$ & $O R^{\star \star \star}$ & $\begin{array}{c}195^{\circ} \\
\text { Inferior }\end{array}$ & $\begin{array}{l}\text { \% IC) } \\
\text { Superior }\end{array}$ \\
\hline Polifarmácia & 1,071 & 0,341 & 9,874 & 1 & 0,002 & 2,920 & 1,497 & 5,696 \\
\hline Polimorbidade & 0,304 & 0,313 & 0,945 & 1 & 0,331 & 1,355 & 0,734 & 2,501 \\
\hline Depressão & 0,620 & 0,299 & 4,297 & 1 & 0,038 & 1,859 & 1,034 & 3,340 \\
\hline
\end{tabular}

A análise multivariada (usando o modelo de regressão logística múltipla) confirmou os resultados da análise univariada, exceto para polimorbidade (Tabela 3), que perdeu significância nesta etapa da análise.

Tabela 3. Análise multivariada das variáveis que influenciaram o uso de MPI

\begin{tabular}{|c|c|c|c|c|c|c|c|c|}
\hline Variáveis & $\begin{array}{l}\text { Coeficiente } \\
\text { (B) }\end{array}$ & $\begin{array}{c}\text { Erro } \\
\text { Padrão }\end{array}$ & Wald & G.L* & p valor ${ }^{* *}$ & $\mathbf{O R}^{\star \star \star *}$ & $\begin{array}{r}\quad 95 \\
\text { Inferior }\end{array}$ & $\begin{array}{l}\text { \% IC) } \\
\text { Superior }\end{array}$ \\
\hline Polifarmácia & 1,071 & 0,341 & 9,874 & 1 & 0,002 & 2,920 & 1,497 & 5,696 \\
\hline Polimorbidade & 0,304 & 0,313 & 0,945 & 1 & 0,331 & 1,355 & 0,734 & 2,501 \\
\hline Depressão & 0,620 & 0,299 & 4,297 & 1 & 0,038 & 1,859 & 1,034 & 3,340 \\
\hline
\end{tabular}

\section{DISCUSSÃO}

Os resultados deste trabalho são relevantes, sobretudo em função da lacuna existente acerca do conhecimento da farmacoterapia em idosos brasileiros institucionalizados. Além de se tratar de um tema ainda pouco conhecido no Brasil, é o primeiro estudo a verificar os fatores que podem influenciar o uso de medicamentos inapropriados para idosos em ILPI do país.

Além disso, como afirma Silva et al. ${ }^{22}$, identificar as características e os fatores associados ao consumo de medicamentos (no caso do presente estudo, especificamente de medicamentos potencialmente impróprios) pelos idosos brasileiros pode auxiliar no planejamento de ações para promoção do uso racional de medicamentos e, consequentemente, favorecer uma melhor qualidade de vida para este grupo etário, além de contribuir para a redução de gastos desnecessários pelo sistema de saúde.

O consumo médio de medicamentos utilizados diariamente pelos idosos do presente estudo, apesar de ser considerado alto, parece ser inferior ao de outros estudos $^{23,24,25}$. Por outro lado, a prevalência do uso de MPI apresentou-se superior aos resultados encontrados por outros autores em ILPI de vários países, como os europeus ${ }^{26}$, o Japão $^{27}$ e até mesmo o Brasil ${ }^{14}$.

Dhala e colaboradores ${ }^{28}$ compararam a prevalência de MPI antes e após a inserção do idoso em ILPI e verificaram que a proporção de pacientes que receberam uma prescrição com pelo menos um MPI caiu de $25,4 \%$ para $20,8 \%$ após a admissão na instituição. Esse estudo ilustrou a alta qualidade da prescrição medicamentosa de casas de repouso para idosos no Canadá, realidade diferente da encontrada no presente estudo.

Essas desigualdades na prevalência do uso de medicamentos podem refletir diferenças entre as populações quanto ao estado de saúde, tipo de serviços de saúde utilizados e modelo de atenção à saúde, além de traços demográficos e culturais diferentes, ligados ao consumo de medicamentos, como explica Loyola Filho et al. ${ }^{29}$.
Entre os idosos que vivem na sociedade, excluindo os institucionalizados, é alta a prevalência de cardiopatias ${ }^{18}$. Por isso, os medicamentos cardiovasculares representam a categoria terapêutica mais utilizada entre eles, conforme alguns estudos ${ }^{30-32}$. Entretanto, a morbidade de idosos que residem em ILPI difere dos idosos não institucionalizados, fato evidenciado no presente estudo, em que os distúrbios mentais figuram como uma das principais patologias.

Consequentemente, o perfil da farmacoterapia dos idosos residentes em ILPI, no tocante às classes terapêuticas mais utilizadas também é diferente dos idosos da comunidade, apresentando uma predominância de medicamentos com ação sobre o sistema nervoso central (36\%), corroborando com os resultados encontrados por Shah et al. ${ }^{33}$, em que o uso de antidepressivos foi superior ao uso de medicação cardiovascular entre idosos institucionalizados da Inglaterra.

Quanto aos fatores de risco relacionados ao uso de MPI, verificou-se que a polifarmácia e a depressão apresentaram significância estatística quanto a essa associação, corroborando com vários outros autores ${ }^{9,19,34,35}$.

Como afirmam Bao e colaboradores ${ }^{35}$, não é de surpreender que a polifarmácia foi um fator fortemente associado ao uso de MPI, visto que pacientes com múltiplos medicamentos em uso tem mais chances de receber uma prescrição inadequada e estão mais propensos a ter múltiplas comorbidades, hospitalizações e consultas com médicos de várias especialidades.

Segundo Wawruch et al. ${ }^{19}$, a associação entre o uso de MPI e certas patologias, como no caso, a depressão, pode ser um importante marcador do incorreto gerenciamento da doença e a tendência de prescrições irracionais.

Apesar de ser um estudo transversal, fato que limita a identificação da relação causa e efeito, a metodologia aplicada não permitiu subnotifcações de medicamentos nem subestimação da sua utilização, visto que a coleta de dados não baseou-se na capacidade dos idosos em se lembrar dos medicamentos que fazem uso, e sim da análise dos prontuários médicos, rigorosamente controlados pelas enfermeiras responsáveis. Assim, a limitação deste trabalho baseia-se no fato de que os resultados desta pesquisa podem não ser representativos para idosos que vivem em ILPI de outras regiões do país, sobretudo nas regiões mais pobres. Logo, é importante que novos estudos sejam realizados, a fim de determinar não só a prevalência da prescrição inadequada, mas o seu impacto na morbimortalidade dos idosos, sobretudo os institucionalizados.

Sugere-se ainda, a importância do profissional no estímulo ao emprego de medidas não-farmacológicas, acompanhamento, com revisão periódica, do conjunto dos medicamentos e seus possíveis efeitos adversos, dando preferência por fármacos de eficácia comprovada através de evidências científicas, sendo os critérios de Beers uma importante ferramenta para avaliar a qualidade da farmacoterapia entre idosos. Todavia, é necessário enfatizar que os critérios devem ser utilizados como guia ao profissional de saúde, visto que o tratamento medicamentoso ao paciente idoso deve ser cuidadosamente individualizado, devendo prevalecer o julgamento clínico, especialmente ao que tange idosos institucionalizados, cujas peculiaridades diferem dos idosos da comunidade.

\section{CONCLUSÃO}

Portanto, este estudo constatou uma alta prevalência de idosos acometidos pela polifarmácia e que utilizavam 
medicamentos potencialmente impróprios (MPI), sendo principalmente administrados fármacos com ação no sistema nervoso central, em consonância com a elevada porcentagem de sujeitos pesquisados com diagnóstico de distúrbios mentais. Também foi verificado que os principais fatores associados ao uso de MPI em idosos institucionalizados foram a polifarmácia e a depressão.

\section{APOIO FINANCEIRO}

À FAPESP - Fundação de Amparo à Pesquisa do Estado de São Paulo, pelo apoio financeiro (auxílio Pesquisa Regular - processo 2011/12525-5) e a CAPES (Coordenação de Aperfeiçoamento de Pessoal de Nível Superior), pelo auxílio referente à bolsa de apoio ao curso de doutoramento de Thaís Jaqueline Vieira de Lima.

\section{REFERÊNCIAS}

1. Soares MAMS. Avaliação terapêutica potencialmente inapropriada no doente geriátrico [tese]. Lisboa: Universidade de Lisboa; 2009.

2. Beers MH, Ouslander JG, Rollingher I, Reuben DB, Brooks J, Beck JC. Explicit criteria for determining inappropriate medication use in nursing home residents. UCLA Division of Geriatric Medicine. Arch Intern Med. 1991;151(9):1825-32.

3. Beers MH. Explicit criteria for determining potentially inappropriate medication use by the elderly.An update. Arch Intern Med. 1997;157(14):1531-6.

4. Fick DM, Cooper JW, Wade WE, Waller JL, Maclean JR, Beers MH. Updating the Beers criteria for potentially inappropriate medication use in older adults: results of a US consensus panel of experts. Arch Inter Med. 2003;163(22):2716-24.

5. Gorzoni ML, Fabri RMA, Pires SL. Critérios de BeersFick e medicamentos genéricos no Brasil. Rev Assoc Med Bras. 2008;54(4):353-6.

6. Resnick B, Pacala JT. 2012 Beers criteria. Journal of the American Geriatrics Society. 2012;60(4):612-3.

7. American Geriatrics Society 2012 Beers Criteria Update Expert Panel. American Geriatrics Society updated Beers Criteria for potentially inappropriate medication use in older adults. J Am Geriatr Soc. 2012; 60(4):616-31.

8. Bongue B, Laroche ML, Gutton S, Colvez A, Guéguen R, Moulin JJ et al. Potentially inappropriate drug prescription in the elderly in France: a population-based study from the French National Insurance Healthcare system. Eur J Clin Pharmacol. 2011;67(12):1291-9.

9. Chen LL, Tangiisuran B, Shafie AA, Hassali MA. Evaluation of potentially inappropriate medications among older residents of Malasyan nursing homes. Int $\mathbf{J}$ Clin Pharm. 2012; 34(4):596-603.

10. Chrischilles EA, VanGilder R, Wright K, Kelly M, Wallace RB. Inappropriate medication use as a risk factor for self-reported adverse drug effects in older adults. J Am Geriatr Soc. 2009; 57(6):1000-6.

11. Klarin I, Fastbom J, Wimo A. A population-based study of drug use in the very old living in a rural district of Sweden, with focus on cardiovascular drug consumption: comparison with an urban cohort. Pharmacoepidemiol Drug Saf. 2003; 12(8):669-78.

12. Dal Pizzol Tda S, Pons Eda S, Hugo FN, Bozzetti MC, Sousa Mda L, Hilgert JB. Uso de medicamentos entre idosos residentes em áreas urbanas e rurais de município do Sul do Brasil: um estudo de base populacional. Cad Saúde Pública. 2012; 28(1):104-14.
13. Simões MJS, Marques AC. Consumo de medicamentos por idosos segundo prescrição médica em Jaú-SP. Rev Ciênc Farm Básica Apl. 2005; 26(2):139-44.

14. Castellar JI, Karnikowski MGO, Vianna LG, Nóbrega OT. Estudo da farmacoterapia prescrita a idosos em instituição brasileira de longa permanência. Acta Med Port. 2007; 20(2):97-105.

15. Morais COM. Avaliação do consumo de medicamentos em instituição asilar [dissertação]. Rio de Janeiro: Universidade Federal do Rio de Janeiro; 1998.

16. Katz S, Downs TD, Cash HR, Grotz RC. Progress in development of the index of ADL. Gerontologist. 1970; $10(1): 20-30$.

17. World Health Organization (WHO). Collaborating Centre for Drug Statistics Methodology. The ATC classification - structure and principles. Disponível em: http://www.whocc.no/atc_ddd_index/. Acesso em: 20 maio 2017.

18. Coelho Filho JM, Marcopito LF, Castelo A Perfil de utilização de medicamentos por idosos em área urbana do Nordeste do Brasil. Rev Saúde Pública. 2004; 38(4):557-64

19. Wawruch M, Fialova D, Zikavska M, Wsolova L, Jezova D, Kuzelova $M$ et al. Factors influencing the use of potentially inappropriate medication in older patients in Slovakia. J Clin Pharm Ther. 2008; 33(4):381-92.

20. Organização Mundial da Saúde (OMS). Classificação estatística internacional de doenças e problemas relacionados à saúde. 10a rev. São Paulo: Centro Colaborador da OMS para a Classificação de Doenças em Português; 1995.

21. Brasil. Ministério da Saúde. Conselho Nacional de Saúde. Resolução n.196, de 10 de outubro de 1996. Dispõe sobre as diretrizes e normas regulamentadoras de pesquisas envolvendo seres humanos. Diário Oficial da República Federativa do Brasil, Brasília (DF); 1996.

22. Silva AL, Ribeiro AQ, Klein CH, Acurcio FA. Utilização de medicamentos por idosos brasileiros, de acordo com a faixa etária: um inquérito postal. Cad Saúde Pública. 2012; 28(6):1033-45.

23. Rajska-Neumann A, Wieczorowska-Tobis K. Polypharmacy and potential inappropriateness of pharmaco-logical treatment among community-dwelling elderly patients. Arch Gerontol Geriatr. 2007;44(Suppl1):303-9.

24. Hosia-Randell HM, Muurinen SM, Pitkälä KH. Exposure to potentially inappropriate drugs and dru-drug interactions in elderly nursing home residents in Helsinki, Finland: a cross-sectional study. Drugs Aging. 2008; 25(8):683-92.

25. Nixdorff N, Hustey FM, Brady AK, Vaji K, Leonard M, Messinger-Rapport BJ. Potentially inappropriate medication and adverse drug effects in elders in the ED. Am J Emerg Med. 2008; 26(6):697-700.

26. Fialová D, Topinková D, Gambassi G, Finne-Soveri H, Jónsson PV, Carpenter I et al. Potentially inappropriate medication use among elderly home care patients in Europe. JAMA. 2005; 293(11):1348-58.

27. Niwata S, Yamada Y, Ikegami N. Prevalence of inappropriate medication using Beers criteria in Japanese long-term care facilities. BMC Geriatr. 2006;6:1.

28. Dhalla IA, Anderson GM, Mandani MM, Bronskill SE, Sykora K, Rochon PA. Inappropriate prescribing before and after nursing home admission. J Am Geriatr Soc. 2002; 50(6):995-1000. 
29. Loyola Filho AI, Uchoa E, Lima-Costa MF. Estudo epidemiológico de base populacional sobre uso de medicamentos entre idosos na Região Metropolitana de Belo Horizonte, Minas Gerais, Brasil. Cad Saúde Pública. 2006; 22(12):2657-67.

30. Bueno CS, Oliveira KR, Berlezi EM, Eickhoff HM, Dallepiane LB, Girardon-Perlini NMO et al. Utilização de medicamentos e risco de interações medicamentosas em idosos atendidos pelo Programa de Atenção ao Idoso da Unijuí. Rev Ciênc Farm Básica Apl. 2009; 30(3):331-8.

31. Flores VB, Benvegnú LA. Perfil de utilização de medicamentos em idosos da zona urbana de Santa Rosa, Rio Grande do Sul, Brasil. Cad Saúde Pública. 2008; 24(6):1439-46.

32. Ribeiro AQ, Rozenfeld S, Klein CH, César CC, Acurcio FA. Inquérito sobre uso de medicamentos por idosos aposentados, Belo Horizonte, MG. Rev Saúde Pública. 2008; 42(4):724-32.

33. Shah SM, Carey IM, Harris T, DeWilde S, Cook DG; Quality of prescribing in care homes and the community in England and Wales. $\mathrm{Br} \mathrm{J}$ Gen Pract. 2012; 62(598):e329-e36.

34. Locatelli J, Lira AR, Torraga LK, Paes AT. Inappropriate medications using the Beers criteria in Brazilian hospitalized elderly patients. Consult Pharm. 2010; 25(1):36-40.

35. Bao Y, Shao H, Bishop T, Schackman BR, Bruce ML. Inappropriate medication in a National Sample of US elderly patients, receiving home health care. J Gen Intern Med. 2012; 27(3):304-10.

\section{CONFLITO DE INTERESSES}

Os autores declaram não haver conflitos de interesse.

\section{AUTOR PARA CORRESPONDÊNCIA}

\section{Clea Adas Saliba Garbin}

cgarbin@foa.unesp.br

Submetido em 26/04/2017

Aceito em 06/06/2017 